\title{
¿S Research Square

\section{Modified FOLFIRINOX as a second-line therapy following gemcitabine plus nab-paclitaxel therapy in metastatic pancreatic cancer}

\section{Masashi Sawada}

Cancer Institute Hospital of Japanese Foundation of Cancer Reseach, Tokyo

Akiyoshi Kasuga ( $\square$ akiyoshi.kasuga@jfcr.or.jp )

Keio University https://orcid.org/0000-0002-2234-1135

Takafumi Mie

Cancer institute Hospital

\section{Takaaki Furukawa}

Cancer Institute Hospital

\section{Takanobu Taniguchi}

Cancer Institute Hospital

\section{Koshiro Fukuda}

Cancer Institute Hospital

\section{Yuto Yamada}

Cancer Institute Hospital

\section{Tsuyoshi Takeda}

Cancer Institute Hospital

\section{Ryo Kanata}

Cancer Institute Hospital

\section{Masato Matsuyama}

Cancer Institute Hospital

\section{Takashi Sasaki}

Cancer Institute Hospital

\section{Masato Ozaka}

Cancer Institute Hospital

\section{Naoki Sasahira}

Cancer Institute Hospital

\section{Research article}

Keywords: pancreatic cancer, second-line chemotherapy, modified FOLFIRINOX, gemcitabine, nabpaclitaxel, prognostic factor 
Posted Date: May 15th, 2020

DOI: https://doi.org/10.21203/rs.3.rs-18686/v3

License: (9) This work is licensed under a Creative Commons Attribution 4.0 International License. Read Full License

Version of Record: A version of this preprint was published at BMC Cancer on May 20th, 2020. See the published version at https://doi.org/10.1186/s12885-020-06945-8. 


\section{Abstract}

Background There is no established second-line treatment after failure of gemcitabine plus nab-paclitaxel $(\mathrm{GnP})$ therapy for metastatic pancreatic cancer (MPC). This study aimed to evaluate the efficacy and tolerability of the modified FOLFIRINOX (mFFX) as a second-line therapy for MPC and investigate prognostic factors for survival.

Methods From 2015-2019, we retrospectively reviewed the medical records of patients receiving mFFX for MPC after failure of GnP therapy. Patients were treated every 2 weeks with $\mathrm{mFFX}$ (intravenous oxaliplatin $85 \mathrm{mg} / \mathrm{m} 2$, intravenous irinotecan $150 \mathrm{mg} / \mathrm{m} \mathrm{2}$, and continuous infusion of 5-fluorouracil $2,400 \mathrm{mg} / \mathrm{m} 2$ for 46 hours without bolus infusion) until disease progression, patient refusal, or unacceptable toxicity.

Results In total, 104 patients received mFFX. The median overall survival (OS) was 7.0 months (95\% confidence interval [Cl]: 6.2-9.8) and the progression-free survival (PFS) 3.9 months (95\% $\mathrm{Cl} 2.8-5.0)$. The objective response rate was $10.6 \%$ and the disease control rate $56.7 \%$. The median relative dose intensities of oxaliplatin, irinotecan, and infusional 5-FU were $80.0 \%$ (range $21.5-100 \%$ ), $77.2 \%$ (range $38.1-100 \%$ ), and $85.9 \%$ (range 36.9-100\%), respectively. Grade 3-4 toxicities were reported in 57 patients (54.8\%), including neutropenia, leukopenia, anemia, febrile neutropenia, and peripheral sensory neuropathy. Glasgow prognostic score and carcinoembryonic antigen level were independently associated with survival. Our prognostic model using these parameters could classify the patients into good $(n=38)$, intermediate $(n=47)$, and poor $(n=19)$ prognostic groups. The median OS and PFS time was 14.7 (95\% $\mathrm{Cl} 7.6-16.3)$ and 7.6 months (95\% Cl 4.1-10.5) for the good prognostic factors, $6.5(95 \% \mathrm{Cl}$ 5.5-10.0) and 3.6 months $(95 \% \mathrm{Cl} 2.7-4.8)$ for the intermediate prognostic factors and $5.0(95 \% \mathrm{Cl} 2.9-6.6)$ and 1.7 months $(95 \% \mathrm{Cl} 0.9-4.3)$ for the poor prognostic factors, respectively.

Conclusions The mFFX showed to be a tolerable second-line treatment for MPC after GnP failure. Our prognostic model might be useful for deciding whether $\mathrm{mFFX}$ is indicated in this setting.

\section{Introduction}

Pancreatic cancer (PC) is the fourth leading cause of cancer-related deaths in the United States and Europe and accounts for $>33,000$ deaths in Japan annually [1]. Although multidisciplinary collaboration and multimodality treatment approaches are important in improving the survival of patients with PC, more than half of patients are diagnosed in the metastatic stage and the prognosis is poor. Conventional cytotoxic chemotherapy remains the standard treatment for patients with metastatic pancreatic cancer (MPC).

Gemcitabine (GEM) alone has long been the standard treatment for MPC [2]. Recently, a combination therapy of FOLFIRINOX (FFX) (5-fluorouracil [5-FU], leucovorin, irinotecan, and oxaliplatin) and GEM plus nab-paclitaxel (GnP) has shown superiority over GEM monotherapy [3,4]. To reduce the severe adverse events of FFX, the use of a modified FFX (mFFX) as a first-line treatment has been evaluated and has 
demonstrated a similar efficacy and reduced toxicity to FFX [5]. This modified regimen is widely used in daily practice in Japan. With the growing number of available drugs for $\mathrm{PC}$, the overall treatment outcomes of chemotherapy for MPC are significantly improved in daily practice [6] [7].

Although several randomized clinical trials have shown survival benefits of second-line chemotherapy after GEM-based therapy, there is no standard second-line chemotherapy regimen for MPC [8] [9] [10]. Moreover, both FFX and GnP have shown better outcomes than GEM alone. Strategies based on FFX followed by GnP, or vice versa, have been tested and used in practice. However, there is limited research about FFX after failure of GnP. This retrospective study aimed to evaluate the efficacy and tolerability of mFFX after failure of first-line GnP in patients with MPC and to clarify the characteristics of patients who will benefit from second-line mFFX.

\section{Methods}

\section{Patients}

Between April 2015 and March 2019, this study enrolled consecutive patients with MPC who received $\mathrm{mFFX}$ after GnP therapy at our hospital. We retrospectively reviewed the medical records of the patients from the prospectively maintained institutional database for PC.

The patient selection criteria were as follows: presence of a pathological and clinical diagnosis of adenocarcinoma; disease progression or intolerance while under GnP chemotherapy; no prior chemotherapy with 5-FU, oxaliplatin, or irinotecan; an Eastern Cooperative Oncology Group performance status (ECOG PS) of 0-2; and adequate bone marrow, renal, and liver functions.

The exclusion criteria were as follows: dose reduction of any drug from the first cycle with the exception of reduction of irinotecan due to a uridine diphosphate glucuronosyltransferase (UGT) 1A1 status as described below; locally advanced pancreatic carcinoma; and severe complications such as active infection, uncontrolled diabetes, massive pleural effusion or ascites, and active concomitant malignancy.

\section{Treatment}

Patients were treated with mFFX every 2 weeks, as follows: a 2-hour intravenous infusion of oxaliplatin $85 \mathrm{mg} / \mathrm{m}^{2}$, a 2-hour intravenous infusion of I-leucovorin $200 \mathrm{mg} / \mathrm{m}^{2}$, a 90-minute intravenous infusion of irinotecan $150 \mathrm{mg} / \mathrm{m}^{2}$, and a continuous 46-hour intravenous infusion of 5-FU $2400 \mathrm{mg} / \mathrm{m}^{2}$, with an omission of bolus 5-FU infusion [5]. All patients routinely received palonosetron $0.75 \mathrm{mg}$ intravenously, dexamethasone $6.6 \mathrm{mg}$ intravenously, and aprepitant $125 \mathrm{mg}$ orally on day 1 , followed by aprepitant 80 $\mathrm{mg}$ orally on days 2 and 3 and dexamethasone $8 \mathrm{mg}$ orally on days 2-4 for antiemetic prophylaxis. Granulocyte-colony stimulating factor was not allowed as the primary prophylaxis. Irinotecan was reduced to $100 \mathrm{mg} / \mathrm{m}^{2}$ for patients with UGT genetic polymorphisms such as homozygous UGT1A1*28 
or UGT1A $1{ }^{*} 6$ and heterozygous UGT1A $1 * 6$ or UGT1A $1 * 28$. For patients with no available results for UGT genetic polymorphism status, irinotecan was started with the initial dose reduced to $100 \mathrm{mg} / \mathrm{m}^{2}$. The dose of any drug was reduced at the discretion of the treating physician, according to the presence of adverse events. The treatment was continued until disease progression, unacceptable toxicity, or patient refusal.

\section{Data collection and evaluation}

Pretreatment evaluation included collection of data on age, sex, ECOG PS, location of PC, disease status, metastatic sites (liver, lung, and peritoneum), and presence of biliary drainage. Laboratory variables such as carbohydrate antigen 19-9 (CA19-9), carcinoembryonic antigen (CEA), albumin (Alb), and C-reactive protein (CRP) levels and neutrophil and lymphocyte counts were initially recorded as continuous variables. Quantitative data were expressed as medians (with ranges), and qualitative data as percentages. The continuous variables were later dichotomized according to the median or reference value of each variable.

We evaluated the relative dose intensity (RDI) of oxaliplatin, irinotecan, and 5-FU. RDI was calculated as the ratio of the actual dose intensity (ADI) to the standard dose intensity (SDI), where ADI was the ratio of the actual dose to the actual duration of chemotherapy, and SDI was the ratio of the standard dose to the standard duration of the regimen. Tumor response was assessed every 2-3 months using contrastenhanced computed tomography, according to the Response Evaluation Criteria in Solid Tumors (RECIST) version 1.1. Progression-free survival (PFS) was counted from the date of treatment initiation to

the date of documentation of disease progression or the last follow-up, while overall survival (OS) was counted from the date of treatment initiation to the date of death or the last follow-up. OS and PFS were calculated using the Kaplan-Meier method. Adverse effects were graded using the National Cancer Institute Common Terminology Criteria for Adverse Events (CTCAE) version 5.0. As inflammation-based prognostic factors, Glasgow prognostic score (GPS) and neutrophil-to-lymphocyte ratio (NLR) were also evaluated [11] [12]. GPS was defined as follows: patients with both an elevated CRP level $(>1.0 \mathrm{mg} / \mathrm{dL})$ and hypoalbuminemia (Alb $<3.5 \mathrm{~g} / \mathrm{dL}$ ) were allocated a score of 2; patients with only one of these biochemical abnormalities were given a score of 1 ; and patients with neither of these abnormalities were scored 0. Clinical data were monitored until May 2019.

\section{Statistical analysis}

Relationships between clinical variables and survival were investigated using univariate and multivariate analyses. The log-rank test was used to evaluate differences in survival. The multivariate analysis was carried out using stepwise Cox proportional hazards regression modeling to identify independent prognostic factors. Each patient was then assigned a prognostic index value, calculated based on the 
number of major independent predictors of survival and weighted using the likelihood ratio of the independent factors. Patients were stratified based on this prognostic index. $p$ values of $<0.05$ were considered statistically significant. All statistical analyses were performed using the SPSS statistical software program (version 20.0; SPSS, Chicago, IL, USA).

\section{Results}

\section{Patient characteristics}

The characteristics of the subjects are shown in Table 1. The study enrolled 104 patients. The median age was 63 years. Among the 104 patients, 75 (72.1\%) were male, 103 (99.0\%) had an ECOG PS of 0-1, $26.0 \%$ had the head of the pancreas as the primary tumor site, $29.8 \%$ experienced recurrence after resection, and $17.3 \%$ had a biliary stent. The major site of metastasis was the liver (69.2\%). UGT genetic polymorphisms were classified as wild-type (46.2\%), heterozygote (44.2\%), double heterozygote (3.8\%), and homozygote (3.8\%), with some patients having no available data $(1.9 \%)$. The median time to failure of $\mathrm{GnP}$ therapy was 194 days. Thirty-one (29.8\%) patients were treated with pregabalin or duloxetine for peripheral sensory neuropathy (PN) induced by nab-paclitaxel. The mFFX therapy was usually started because of disease progression (97\%) and intolerance (3\%).

\section{Toxicity}

Adverse events are summarized in Table 2. Grade 3-4 toxicities occurred in 57 patients (54.8\%). The major grade 3-4 hematological toxicities were neutropenia (42.3\%), leucopenia (24.0\%), and anemia (17.3\%). The incidence of febrile neutropenia was 5.8\%. The major grade 3-4 nonhematological toxicities were PN (10.6\%), diarrhea (2.9\%), and anorexia (1.0\%). Interstitial pneumonia occurred in one patient (1.0\%). There were no treatment-related deaths.

\section{Treatment exposure}

The median follow-up time was 188 days (range 14-707). A total of 883 cycles were delivered to the 104 patients. The median number of treatment cycles was 6 (range 1-47). The median RDIs of oxaliplatin, irinotecan, and infusional 5-FU were $80.0 \%$ (range $21.5-100 \%$ ), $77.2 \%$ (range $38.1-100 \%$ ), and $85.9 \%$ (range $36.9-100 \%)$, respectively. Dose reduction occurred in 75 patients $(72.1 \%)$, and treatment delay in 71 patients $(68.2 \%)$. Neutropenia was the most frequent cause of both dose reduction and treatment delay. Oxaliplatin treatment was suspended in 18 patients (17.3\%). The reasons for treatment suspension were PN in 10 patients $(79.8 \%)$, allergy in $3(2.9 \%)$, and others in 5 . The median number of cycles of $\mathrm{mFFX}$ before the suspension of oxaliplatin due to PN was 12 (range 3-18). On the other hand, the reasons for treatment discontinuation were disease progression in 83 patients $(79.8 \%)$, adverse events in $2(1.9 \%)$, and transfer to another hospital in $2(1.9 \%)$. Thus, 17 patients were on treatment. 


\section{Efficacy}

Eleven (10.6\%) patients showed partial responses, while $48(46.2 \%)$ showed stable disease, resulting in a disease control rate of $56.7 \%$. The median OS time was 7.0 months (95\% confidence interval [CI] 6.2-9.8), and the median PFS time was 3.9 months ( $95 \% \mathrm{Cl}$ 2.8-5.0) (Fig. 1). The median OS time after the start of the first-line therapy was 15.9 months $(95 \% \mathrm{Cl} 13.5-18.8)$.

After disease progression on $\mathrm{mFFX}$ therapy, 34 patients (32.7\%) received a third line of chemotherapy, as follows: 14 patients (13.5\%) received GEM plus erlotinib, 13 (12.5\%) received S-1 monotherapy, and 7 $(6.7 \%)$ received other regimens.

\section{Prognostic factors}

The median survival time and $p$ values for the univariate analysis are shown in Table 3. Among the variables, ECOG PS $=0$, CEA level $\leq 10 \mathrm{ng} / \mathrm{mL}$, Alb $\geq 3.5 \mathrm{~g} / \mathrm{dL}, \mathrm{NLR} \leq 5.0, \mathrm{CRP} \leq 0.16 \mathrm{~g} / \mathrm{dL}$, and GPS $=0$ were significantly associated with longer survival. CA19-9 level was not a prognostic factor.

The results of the Cox proportional hazards model are shown in Table 4. In the multivariate analysis, CEA level $\leq 10 \mathrm{ng} / \mathrm{mL}$ and GPS $=0$ were identified as independent prognostic factors. For the clinical application of these findings, a prognostic index was calculated. One point was assigned for each variable and added for a composite score of $0-2$. The patients were then assigned to three subgroups according to their prognostic index, as follows: good prognostic group, prognostic index $=0(n=38)$; intermediate prognostic group, prognostic index $=1(n=47)$; and poor prognostic group, prognostic index $=2(n=19)$. The median OS time was 14.7 months ( $95 \%$ Cl $7.6-16.3)$ in the good prognostic group, 6.5 months (95\% Cl 5.5-10.0) in the intermediate prognostic group, and 5.0 months (95\% $\mathrm{Cl} 2.9-6.6)$ in the poor prognostic group (good vs intermediate, $p<0.05$; intermediate vs poor, $p<0.01$ ) (Fig. 2a). The median PFS time was 7.6 months $(95 \% \mathrm{Cl} 4.1-10.5)$ in the good prognostic group, 3.6 months $(95 \% \mathrm{Cl}$ 2.7-4.8) in the intermediate prognostic group, and 1.7 months ( $95 \% \mathrm{Cl} 0.9-4.3)$ in the poor prognostic group (good vs intermediate, $p<0.01$; intermediate vs poor, $p<0.05$ ) (Fig. 2b). Our prognostic model was able to classify the patients into three groups with significantly different values for PFS and OS.

\section{Discussion}

This retrospective study investigated mFFX therapy in patients with GnP-refractory MPC. The patients demonstrated an objective response rate of $10.6 \%$, disease control rate of $56.7 \%$, and median PFS and OS of 3.9 and 7.0 months, respectively. The median OS time after the start of the first-line therapy was 15.9 months. After exploring the independent variables associated with survival in this setting, we identified the CEA level and GPS as independent prognostic factors. This study also showed that the 
median PFS and OS of patients in good general condition with these good prognostic factors were 7.6 and 14.7 months, respectively.

Regarding second-line chemotherapy for GEM-refractory PC, a prematurely discontinued randomized clinical trial (RCT) by the German CONKO-study group provided evidence on the benefit of second-line chemotherapy compared with best supportive care [9]. In a recent NAPOLI-1 phase III trial, significant improvements in both PFS and OS were observed in patients with MPC after previous GEM-based therapy and irinotecan liposomal injection combined with 5-FU/l-leucovorin, indicating a new treatment option for this population [10]. The NAPOLI-1 study showed a median PFS and OS of 3.1 and 6.1 months, respectively, for a combination of 5-FU and liposomal irinotecan. Oxaliplatin combination therapy in this setting has also been investigated by three RCTs, with different and confounding results (CONKO-003, PANCREOX, and SOX [S-1 plus oxaliplatin]) [13] [14]. In a recent large phase III GRAPE trial comparing S-1 plus leucovorin and S-1 in patients with a good performance status and Alb $\geq 3.5 \mathrm{~g} / \mathrm{dL}$, the median PFS and OS were 2.8 and 7.9 months, respectively [15]. Meanwhile, in our study, the median PFS and OS for patients with Alb $\geq 3.5 \mathrm{~g} / \mathrm{dL}$ were 5.1 and 9.5 months, respectively. Sequential therapies with FFX and $\mathrm{GnP}$ have been tested because FFX and GnP are more effective as first-line therapy than GEM alone. A prospective cohort study in France evaluated second-line GnP after first-line FFX in 57 patients and showed a median PFS and OS of 5.1 months and 8.8 months, respectively, and a median OS of 18 months since the start of first-line FFX [16]. Although comparisons among these previous studies are difficult, their findings led us to suggest that mFFX in selected patients may be effective and promising as a second-line treatment after failure of GnP.

As the FFX regimen has demonstrated better survival benefits than GEM as a first-line treatment, several prospective and retrospective studies evaluated FFX after failure of GEM-based chemotherapy, demonstrating median PFS time and OS times of 2.8-5.8 and 8.4-9.8 months [17] [18] [19] [20]. These results suggest the promising clinical efficacy of the FFX regimen as a second-line treatment. Because FFX is a potentially highly toxic combination of drugs with serious side effects, two of the above studies, which are prospective phase II trial conducted by two Korean groups, reduced the dosage of both irinotecan (120-135 mg/m²) and oxaliplatin $\left(60-65 \mathrm{mg} / \mathrm{m}^{2}\right)$. Despite the dose reduction, these studies showed a promising efficacy of the regimen for patients with PC after failure of GEM-based chemotherapy. In these studies, most of the patients received GEM monotherapy or GEM plus erlotinib as a first-line treatment. In our study, we evaluated the efficacy and tolerability of mFFX after failure of firstline $\mathrm{GnP}$ and found the RDIs of oxaliplatin and irinotecan to be $80.0 \%$ and $77.2 \%$, respectively. Prospective studies are needed to better define the doses of mFFX and to determine the efficacy and risks of mFFX after failure of GnP.

Our study also found that grade $3-4$ toxicities occurred in 57 patients (54.8\%) and consisted mainly of hematological adverse effects (grade 3-4 neutropenia, $42.3 \%$; febrile neutropenia, 5.8\%). Although these incidences of hematologic toxicities are relatively higher than those reported for second-line chemotherapy regimens in previous studies such as the NAPOLI-1 and GRAPE trials and in the previous prospective French cohort treated with GnP after FFX, they are comparable with those reported for mFFX 
as first-line chemotherapy in Japan. In our study, most episodes of the adverse events were reversible, and the treatment was generally well tolerated. Dose reduction and cycle delay were required in about $70 \%$ of the patients in this setting. Moreover, the RDI was relatively low compared with that of mFFX as a first-line treatment in a phase II study in Japan [5]. Although the safety profile in our study suggests that mFFX can be safely used after failure of GnP, the dosage and treatment schedule of mFFX in this setting should be more carefully evaluated in the future. Indeed, several prospective studies to evaluate FFX after failure of GEM-based therapy have reduced the initial dose of irinotecan and oxaliplatin.

One of the main concerns with sequential therapies consisting of GnP and mFFX is the risk of severe chronic PN owing to the use of nab-paclitaxel after oxaliplatin. In this study, the incidence of grade 1-2 PN was $78.8 \%$, and that of grade $3-4$ PN was $10.6 \%$. This toxicity rate is higher than that of FFX or mFFX as first-line chemotherapy in Japan [21] [5]. However, this rate is consistent with that of FFX in the ACCORD11 trial or in the previous prospective French cohort treated with GnP after FFX, and is quite lower than that in the MPACT trial [3] [16] [4]. The mechanisms of oxaliplatin and nab-paclitaxel neurotoxicity are different, and so are their mechanisms of reversibility. Nab-paclitaxel causes paresthesia and distal burning pain, but the symptoms generally improve within 2 months after treatment cessation [22]. Oxaliplatin causes cumulative symmetric paresthesia and distal sensory decline [23]. Therefore, despite an equivalence in toxicity grade by both drugs, the impact of oxaliplatin neurotoxicity on patients' quality of life (QOL) may be less severe. This notion is supported by the finding that patients treated with a combination of FOLFOX and nab-paclitaxel did not develop significant neuropathy when they received fewer than 10 cycles, which is consistent with the occurrence of neuropathy in patients treated with FOLFOX alone [24]. As for nab-paclitaxel-induced PN, the MPACT trial revealed that the median time to improvement from grade 3 to grade 2 was 21 days and that from grade 3 to grade 1 or resolution of the event was 29 days. With a median of only 6 cycles of mFFX per patient in our study, the risk of severe neurotoxicity seems limited in this study.

Subgroup analysis of the patients in this study showed that a low GPS and a low CEA level were independently associated with a good outcome. Previous reports indicated that the performance status, CA19-9 level, duration of the first-line chemotherapy, and inflammation-based prognostic score were important prognostic factors in a salvage setting [25,26] [11] [12] [27] [28] [29]. It is now widely accepted that the inflammation-based prognostic score, such as the GPS, is a reliable indicator of survival for many types of malignant solid tumors including PC in various settings [11] [30]. Tumor markers such as CA19-9 and CEA levels were also reported as important prognostic factors in patients with advanced PC treated with chemotherapy. In our study, although the CA19-9 level and survival did not show a significant difference, patients in this setting with high CEA levels might indicate a more aggressive tumor biology. The question of whether the benefits of mFFX extend to patients even after failure of GnP is relevant because both mFFX and GnP are more intensive than GEM alone and there is no established second-line chemotherapy regimen for PC. Our prognostic model using GPS and CEA levels classified the patients into three prognostic groups (good, intermediate, and poor) and enabled stratification of patients according to both PFS and OS. The median OS times in the poor, intermediate, and good prognostic group were 5.0, 6.5, and 14.7 months, respectively. These results suggest that $\mathrm{mFFX}$ after failure of $\mathrm{GnP}$ 
offered no survival benefits to the poor prognostic group. Thus, the model might be useful for deciding whether $\mathrm{mFFX}$ is indicated for the patients with MPC in this setting.

This study has a few limitations. First, we only performed a single-center retrospective analysis, although the sample size exceeded 100 patients. Second, the patients who received treatment might have been more fit, better able to tolerate the treatment, and therefore more likely to benefit from it. Additionally, the gap between the median OS time and median PFS time in the good prognostic group was relatively large. In the good prognostic group, $28 \%$ of patients received chemotherapy after failure of second-line mFFX. In a previous meta-analysis, post-progression survival following second-line chemotherapy in patients with advanced PC previously treated with GEM was associated with subsequent chemotherapy and supportive care [31]. The subsequent treatment and bias from selecting patients with a good general condition may explain these findings. Lastly, this study did not evaluate biomarkers and QOL. QOL is an important element of palliative chemotherapy for PC. Hence, future prospective studies of second-line mFFX following GnP are necessary to clarify treatment efficacy and QOL of patients in this context.

\section{Conclusions}

mFFX showed to be a tolerable second-line treatment for selected patients with MPC after failure of GnP therapy. Our prognostic model using GPS and CEA level might be useful for deciding whether mFFX is indicated in this setting. These findings need to be confirmed in a comparative randomized trial.

\section{Abbreviations}

ADI: Actual dose intensity; Alb: Albumin; CA19-9: Carbohydrate antigen 19-9; CEA: Carcinoembryonic antigen; $\mathrm{Cl}$ : Confidential interval; CRP: $\mathrm{C}$ reactive protein; CTCAE: Common terminology criteria of adverse events; ECOG: Eastern Cooperative Oncology Group; FFX: FOLFIRINOX; GEM: Gemcitabine; GnP: Gemcitabine plus nab-paclitaxel; GPS: Glasgow prognostic scale; mFFX: modified FOLFIRINOX; MPC: metastatic pancreatic cancer; NLR: Neutrophil-to-lymphocyte ratio; OS: Overall survival; PC: Pancreatic cancer; PFS: Progression free survival; PN: Peripheral neuropathy; PS: Performance status; RCT:

Randomized controlled trial; RDI: Relative dose intensity; RECIST: Response Evaluation Criteria in Solid Tumors; SDI: Standard dose intensity; UGT1A1: Uridine diphosphate glucuronosyltransferase 1A1

\section{Declarations}

Ethical approval and consent to participate: This study was approved by the Institutional Review Board of the Cancer Institute Hospital of Japanese Foundation for Cancer Research (No. 2018-1208) and was conducted in accordance with the Declaration of Helsinki. All patients provided written informed consent.

Consent for publication: Not applicable 
Availability of data and materials: All the data and materials supporting the conclusions were included in the main paper. The datasets used in the current study could be available from the corresponding author on request.

Competing interests: All authors declare no conflicts of interests for this article. Conflicts of interests outside this work are as follows; Naoki Sasahira received honoraria from Toshiba, Cook Medical and Boston Scientific and research funding from Zeria, Eisai, Kyowa Hakko Kirin, Baxalta and Taiho Pharmaceutical. Masato Ozaka received honoraria from Taiho Pharmaceutical, Yakult, Bayer, Pfizer, Novartis, Takeda Pharmaceutical Company Limited, Eisai, EA pharma and Mitsubishi Tanabe Pharma Corporation and research funding from Merck \& Co., Inc., Incyte, ASLAN Pharmaceuticals, Taiho Pharmaceutical and Yakult. Takashi Sasaki received honoraria from Taiho Pharmaceutical, Eisai, Boston Scientific Japan, Century Medical Inc. and Cook Japan.

Funding: This study was not funded.

Authors' contribution: MS and AK contributed equally to this work. MS and AK designed the study. MS and $A K$ acquired the patient data. MS and $A K$ analyzed the data. MS, AK, TM, TF, TT, KF, YY, TT, RK, MM, TS, MO and NS participated in the interpretation of data and drafted the manuscript. All authors read and approved the final manuscript.

Acknowledgements: The authors wish to thank Kikuko Kaji for technical support.

Authors' information: ${ }^{1}$ Department of Hepato-Biliary-Pancreatic Medicine, Cancer Institute Hospital of Japanese Foundation for Cancer Research, Tokyo, Japan

${ }^{2}$ Division of Gastroenterology and Hepatology, Department of Internal Medicine, School of Medicine, Keio University, Tokyo

\section{References}

1. Siegel RL, Miller KD, Jemal A (2017) Cancer Statistics, 2017. CA: a cancer journal for clinicians 67 (1):7-30. doi:10.3322/caac.21387 
2. Burris HA, 3rd, Moore MJ, Andersen J, Green MR, Rothenberg ML, Modiano MR, Cripps MC, Portenoy RK, Storniolo AM, Tarassoff P, Nelson R, Dorr FA, Stephens CD, Von Hoff DD (1997) Improvements in survival and clinical benefit with gemcitabine as first-line therapy for patients with advanced pancreas cancer: a randomized trial. Journal of clinical oncology : official journal of the American Society of Clinical Oncology 15 (6):2403-2413. doi:10.1200/jco.1997.15.6.2403

3. Conroy T, Desseigne F, Ychou M, Bouche O, Guimbaud R, Becouarn Y, Adenis A, Raoul JL, GourgouBourgade S, de la Fouchardiere C, Bennouna J, Bachet JB, Khemissa-Akouz F, Pere-Verge D, Delbaldo C, Assenat E, Chauffert B, Michel P, Montoto-Grillot C, Ducreux M (2011) FOLFIRINOX versus gemcitabine for metastatic pancreatic cancer. The New England journal of medicine 364 (19):18171825. doi:10.1056/NEJMoa1011923

4. Von Hoff DD, Ervin T, Arena FP, Chiorean EG, Infante J, Moore M, Seay T, Tjulandin SA, Ma WW, Saleh MN, Harris M, Reni M, Dowden S, Laheru D, Bahary N, Ramanathan RK, Tabernero J, Hidalgo M, Goldstein D, Van Cutsem E, Wei X, Iglesias J, Renschler MF (2013) Increased survival in pancreatic cancer with nab-paclitaxel plus gemcitabine. The New England journal of medicine 369 (18):16911703. doi:10.1056/NEJMoa1304369

5. Ozaka M, Ishii H, Sato T, Ueno M, Ikeda M, Uesugi K, Sata N, Miyashita K, Mizuno N, Tsuji K, Okusaka T, Furuse J (2018) A phase II study of modified FOLFIRINOX for chemotherapy-naive patients with metastatic pancreatic cancer. Cancer chemotherapy and pharmacology 81 (6):1017-1023. doi:10.1007/s00280-018-3577-9

6. Sasaki T, Kanata R, Yamada I, Matsuyama M, Ozaka M, Sasahira N (2019) Improvement of Treatment Outcomes for Metastatic Pancreatic Cancer: A Real-world Data Analysis. In vivo (Athens, Greece) 33 (1):271-276. doi:10.21873/invivo.11471

7. Kasuga A, Hamamoto Y, Takeuchi A, Kawasaki K, Suzuki T, Hirata K, Sukawa Y, Takaishi H, Kanai T (2017) Positive relationship between subsequent chemotherapy and overall survival in pancreatic cancer: meta-analysis of postprogression survival for first-line chemotherapy. Cancer chemotherapy and pharmacology 79 (3):595-602. doi:10.1007/s00280-017-3263-3

8. Pelzer U, Schwaner I, Stieler J, Adler M, Seraphin J, Dorken B, Riess H, Oettle H (2011) Best supportive care (BSC) versus oxaliplatin, folinic acid and 5-fluorouracil (OFF) plus BSC in patients for secondline advanced pancreatic cancer: a phase III-study from the German CONKO-study group. European journal of cancer (Oxford, England : 1990) 47 (11):1676-1681. doi:10.1016/j.ejca.2011.04.011

9. Oettle H, Riess H, Stieler JM, Heil G, Schwaner I, Seraphin J, Gorner M, Molle M, Greten TF, Lakner V, Bischoff S, Sinn M, Dorken B, Pelzer U (2014) Second-line oxaliplatin, folinic acid, and fluorouracil versus folinic acid and fluorouracil alone for gemcitabine-refractory pancreatic cancer: outcomes from the CONKO-003 trial. Journal of clinical oncology : official journal of the American Society of Clinical Oncology 32 (23):2423-2429. doi:10.1200/jco.2013.53.6995

10. Wang-Gillam A, Li CP, Bodoky G, Dean A, Shan YS, Jameson G, Macarulla T, Lee KH, Cunningham D, Blanc JF, Hubner RA, Chiu CF, Schwartsmann G, Siveke JT, Braiteh F, Moyo V, Belanger B, Dhindsa N, Bayever E, Von Hoff DD, Chen LT (2016) Nanoliposomal irinotecan with fluorouracil and folinic acid in metastatic pancreatic cancer after previous gemcitabine-based therapy (NAPOLI-1): a global, 
randomised, open-label, phase 3 trial. Lancet (London, England) 387 (10018):545-557. doi:10.1016/s0140-6736(15)00986-1

11. Proctor MJ, Morrison DS, Talwar D, Balmer SM, O'Reilly DS, Foulis AK, Horgan PG, McMillan DC (2011) An inflammation-based prognostic score (mGPS) predicts cancer survival independent of tumour site: a Glasgow Inflammation Outcome Study. British journal of cancer 104 (4):726-734. doi:10.1038/sj.bjc.6606087

12. Stotz M, Gerger A, Eisner F, Szkandera J, Loibner H, Ress AL, Kornprat P, AlZoughbi W, Seggewies FS, Lackner C, Stojakovic T, Samonigg H, Hoefler G, Pichler M (2013) Increased neutrophil-lymphocyte ratio is a poor prognostic factor in patients with primary operable and inoperable pancreatic cancer. British journal of cancer 109 (2):416-421. doi:10.1038/bjc.2013.332

13. Gill S, Ko YJ, Cripps C, Beaudoin A, Dhesy-Thind S, Zulfiqar M, Zalewski P, Do T, Cano P, Lam WYH, Dowden S, Grassin H, Stewart J, Moore M (2016) PANCREOX: A Randomized Phase III Study of Fluorouracil/Leucovorin With or Without Oxaliplatin for Second-Line Advanced Pancreatic Cancer in Patients Who Have Received Gemcitabine-Based Chemotherapy. Journal of clinical oncology : official journal of the American Society of Clinical Oncology 34 (32):3914-3920. doi:10.1200/jco.2016.68.5776

14. Ohkawa S, Okusaka T, Isayama H, Fukutomi A, Yamaguchi K, Ikeda M, Funakoshi A, Nagase M, Hamamoto Y, Nakamori S, Tsuchiya Y, Baba H, Ishii H, Omuro Y, Sho M, Matsumoto S, Yamada N, Yanagimoto H, Unno M, Ichikawa Y, Takahashi S, Watanabe G, Wakabayashi G, Egawa N, Tsuda M, Hosotani R, Hamada C, Hyodo I (2015) Randomised phase II trial of S-1 plus oxaliplatin vs S-1 in patients with gemcitabine-refractory pancreatic cancer. British journal of cancer 112 (9):1428-1434. doi:10.1038/bjc. 2015.103

15. loka T, Ueno M, Ueno H, Park JO, Chang HM, Sasahira N, Kanai M, Chung IJ, Ikeda M, Nakamori S, Mizuno N, Omuro Y, Yamaguchi T, Hara H, Sugimori K, Furuse J, Maguchi H, Furukawa M, Fukuzawa K, Kim JS, Yukisawa S, Takeuchi M, Okusaka T, Boku N, Hyodo I (2019) TAS-118 (S-1 plus leucovorin) versus S-1 in patients with gemcitabine-refractory advanced pancreatic cancer: a randomised, open-label, phase 3 study (GRAPE trial). European journal of cancer (Oxford, England : 1990) 106:78-88. doi:10.1016/j.ejca.2018.10.004

16. Portal A, Pernot S, Tougeron D, Arbaud C, Bidault AT, de la Fouchardiere C, Hammel P, Lecomte T, Dreanic J, Coriat R, Bachet JB, Dubreuil O, Marthey L, Dahan L, Tchoundjeu B, Locher C, Lepere C, Bonnetain F, Taieb J (2015) Nab-paclitaxel plus gemcitabine for metastatic pancreatic adenocarcinoma after Folfirinox failure: an AGEO prospective multicentre cohort. British journal of cancer 113 (7):989-995. doi:10.1038/bjc.2015.328

17. Assaf E, Verlinde-Carvalho M, Delbaldo C, Grenier J, Sellam Z, Pouessel D, Bouaita L, Baumgaertner I, Sobhani I, Tayar C, Paul M, Culine S (2011) 5-fluorouracil/leucovorin combined with irinotecan and oxaliplatin (FOLFIRINOX) as second-line chemotherapy in patients with metastatic pancreatic adenocarcinoma. Oncology 80 (5-6):301-306. doi:10.1159/000329803

18. Kobayashi N, Shimamura T, Tokuhisa M, Goto A, Endo I, Ichikawa Y (2017) Effect of FOLFIRINOX as second-line chemotherapy for metastatic pancreatic cancer after gemcitabine-based chemotherapy 
failure. Medicine 96 (19):e6769. doi:10.1097/md.0000000000006769

19. Kim JH, Lee SC, Oh SY, Song SY, Lee N, Nam EM, Lee S, Hwang IG, Lee HR, Lee KT, Bae SB, Kim HJ, Jang JS, Lim DH, Lee HW, Kang SY, Kang JH (2018) Attenuated FOLFIRINOX in the salvage treatment of gemcitabine-refractory advanced pancreatic cancer: a phase II study. Cancer communications (London, England) 38 (1):32. doi:10.1186/s40880-018-0304-1

20. Chung MJ, Kang H, Kim HG, Hyun JJ, Lee JK, Lee KH, Noh MH, Kang DH, Lee SH, Bang S (2018) Multicenter phase II trial of modified FOLFIRINOX in gemcitabine-refractory pancreatic cancer. World journal of gastrointestinal oncology 10 (12):505-515. doi:10.4251/wjgo.v10.i12.505

21. Okusaka T, Ikeda M, Fukutomi A, loka T, Furuse J, Ohkawa S, Isayama H, Boku N (2014) Phase II study of FOLFIRINOX for chemotherapy-naive Japanese patients with metastatic pancreatic cancer. Cancer science 105 (10):1321-1326. doi:10.1111/cas.12501

22. Scripture CD, Figg WD, Sparreboom A (2006) Peripheral neuropathy induced by paclitaxel: recent insights and future perspectives. Current neuropharmacology 4 (2):165-172. doi:10.2174/157015906776359568

23. Starobova H, Vetter I (2017) Pathophysiology of Chemotherapy-Induced Peripheral Neuropathy. Frontiers in molecular neuroscience 10:174. doi:10.3389/fnmol.2017.00174

24. Safran H, Charpentier KP, Perez K, Mantripragada K, Miner T, DiPetrillo T, Kuritzky B, Apor E, Bishop K, Luppe D, Mitchell K, Rosati K (2016) FOLFOX+Nab-Paclitaxel (FOLFOX-A) for Advanced Pancreatic Cancer: A Brown University Oncology Research Group Phase I Study. American journal of clinical oncology 39 (6):619-622. doi:10.1097/coc.0000000000000246

25. Vienot A, Beinse G, Louvet C, de Mestier L, Meurisse A, Fein F, Heyd B, Cleau D, d'Engremont C, Dupont-Gossart AC, Lakkis Z, Tournigand C, Bouche O, Rousseau B, Neuzillet C, Bonnetain F, Borg C, Vernerey D (2017) Overall Survival Prediction and Usefulness of Second-Line Chemotherapy in Advanced Pancreatic Adenocarcinoma. Journal of the National Cancer Institute 109 (10). doi:10.1093/jnci/djx037

26. Tabernero J, Chiorean EG, Infante JR, Hingorani SR, Ganju V, Weekes C, Scheithauer W, Ramanathan RK, Goldstein D, Penenberg DN, Romano A, Ferrara S, Von Hoff DD (2015) Prognostic factors of survival in a randomized phase III trial (MPACT) of weekly nab-paclitaxel plus gemcitabine versus gemcitabine alone in patients with metastatic pancreatic cancer. The oncologist 20 (2):143-150. doi:10.1634/theoncologist.2014-0394

27. Herrmann C, Abel U, Stremmel W, Jaeger D, Herrmann T (2007) Short time to progression under firstline chemotherapy is a negative prognostic factor for time to progression and residual survival under second-line chemotherapy in advanced pancreatic cancer. Oncology 73 (5-6):335-339. doi:10.1159/000134477

28. Sinn M, Dalken L, Striefler JK, Bischoff S, Schweitzer N, Pelzer U, Dorken B, Riess H, Stieler JM (2016) Second-Line Treatment in Pancreatic Cancer Patients: Who Profits?--Results From the CONKO Study Group. Pancreas 45 (4):601-605. doi:10.1097/mpa.0000000000000533 
29. Ziske C, Schlie C, Gorschluter M, Glasmacher A, Mey U, Strehl J, Sauerbruch T, Schmidt-Wolf IG (2003) Prognostic value of CA 19-9 levels in patients with inoperable adenocarcinoma of the pancreas treated with gemcitabine. British journal of cancer 89 (8):1413-1417. doi:10.1038/sj.bjc.6601263

30. Kasuga A, Okano N, Naruge D, Kitamura H, Takasu A, Nagashima F, Furuse J (2015) Retrospective analysis of fixed dose rate infusion of gemcitabine and S-1 combination therapy (FGS) as salvage chemotherapy in patients with gemcitabine-refractory advanced pancreatic cancer: inflammationbased prognostic score predicts survival. Cancer chemotherapy and pharmacology 75 (3):457-464. doi:10.1007/s00280-014-2665-8

31. Kasuga A, Hamamoto Y, Takeuchi A, Okano N, Togasaki K, Aoki Y, Suzuki T, Kawasaki K, Hirata K, Sukawa Y, Kanai T, Takaishi H (2018) Post-progression survival following second-line chemotherapy in patients with advanced pancreatic cancer previously treated with gemcitabine: a meta-analysis. Investigational new drugs 36 (5):939-948. doi:10.1007/s10637-018-0589-6

\section{Tables}

Table 1 Patient characteristics 


\begin{tabular}{|c|c|c|}
\hline Characteristic & & $\%$ \\
\hline \multicolumn{3}{|l|}{ Age (years) } \\
\hline Median & 63 & \\
\hline Range & $37-77$ & \\
\hline \multicolumn{3}{|l|}{ Sex } \\
\hline Male & 75 & 72.1 \\
\hline Female & 29 & 27.9 \\
\hline \multicolumn{3}{|l|}{ ECOG PS } \\
\hline 0 & 93 & 89.4 \\
\hline 1 & 10 & 9.6 \\
\hline 2 & 1 & 1 \\
\hline \multicolumn{3}{|l|}{ Location of pancreatic tumor } \\
\hline Head & 27 & 26 \\
\hline Body/tail & 46 & 44.2 \\
\hline Recurrent after resection & 31 & 29.8 \\
\hline \multicolumn{3}{|l|}{ Metastatic site } \\
\hline Liver & 72 & 69.2 \\
\hline Lung & 19 & 18.3 \\
\hline Peritoneum & 16 & 15.4 \\
\hline \multicolumn{3}{|l|}{ Biliary drainage } \\
\hline Yes & 18 & 17.3 \\
\hline No & 86 & 82.7 \\
\hline \multicolumn{3}{|l|}{ UGT1A1*6/UGT1A1*28 } \\
\hline Wild/wild & 48 & 46.2 \\
\hline Wild/heterozygous & 13 & 12.5 \\
\hline Heterozygous/wild & 33 & 31.7 \\
\hline Heterozygous/heterozygous & 4 & 3.8 \\
\hline Homozygous $(* 6$ or $* 28)$ & 4 & 3.8 \\
\hline NA & 2 & 1.9 \\
\hline \multicolumn{3}{|l|}{ CEA (ng/mL) } \\
\hline Median & 6.1 & \\
\hline Range & $1.2-10068$ & \\
\hline \multicolumn{3}{|l|}{ CA19-9 (IU/mL) } \\
\hline Median & 814.5 & \\
\hline Range & $2-50000$ & \\
\hline \multicolumn{3}{|l|}{ Alb (g/dL) } \\
\hline Median & 3.6 & \\
\hline Range & $2.5-4.4$ & \\
\hline \multicolumn{3}{|l|}{$\mathrm{CRP}(\mathrm{mg} / \mathrm{dL})$} \\
\hline Median & 0.31 & \\
\hline Range & $0.01-8.75$ & \\
\hline
\end{tabular}

Alb albumin, CA19-9 carbohydrate antigen 19-9, CEA carcinoembryonic antigen, CRP C-reactive protein, ECOG PS Eastern Cooperative Oncology Group performance status, NA not available, UGT1A1 uridine diphosphate glucuronosyltransferase 1A1

Table 2 Adverse events according to the Common Terminology Criteria for Adverse Events (CTCAE) version 5.0 


\begin{tabular}{|c|c|c|c|c|c|}
\hline \multirow{2}{*}{$\begin{array}{l}\text { Toxicity } \\
\text { Hematologic }\end{array}$} & All grades & & \multicolumn{3}{|c|}{ Grade 3 or higher } \\
\hline & & & & & \\
\hline Anemia & ( $97.1 \%$ & ) & 18 & $17.3 \%$ & ) \\
\hline Neutropenia & $66.3 \%$ & ) & 44 & $42.3 \%$ & ) \\
\hline Leukopenia & ( $66.3 \%$ & ) & 25 & $24.0 \%$ & ) \\
\hline Thrombocytopenia & ( $70.2 \%$ & ) & 2 & $1.9 \%$ & ) \\
\hline Nonhematologic & & & & & \\
\hline Febrile neutropenia & & & 6 & $5.8 \%$ & ) \\
\hline Anorexia & ( $41.3 \%$ & ) & 1 & $1.0 \%$ & ) \\
\hline Fatigue & ( $85.6 \%$ & ) & 0 & $0.0 \%$ & ) \\
\hline Stomatitis & ( $30.8 \%$ & ) & 0 & $0.0 \%$ & ) \\
\hline Diarrhea & ( $60.6 \%$ & ) & 3 & $2.9 \%$ & ) \\
\hline Constipation & ( $48.1 \%$ & ) & 0 & $0.0 \%$ & ) \\
\hline Nausea & $(68.3 \%$ & ) & 0 & $0.0 \%$ & ) \\
\hline $\mathrm{PN}$ & $89.4 \%$ & ) & 11 & $10.6 \%$ & ) \\
\hline Edema in limbs & ( $27.9 \%$ & ) & 0 & $0.0 \%$ & ) \\
\hline
\end{tabular}

$P N$ peripheral sensory neuropathy

Table 3 Univariate analysis of prognostic factors 


\begin{tabular}{|c|c|c|c|c|}
\hline Sex & & & & \\
\hline & Male & 75 & 6.5 & 0.052 \\
\hline & Female & 29 & 10.6 & \\
\hline ECO & & & & \\
\hline & 0 & 93 & 7.3 & 0.038 \\
\hline & 1,2 & 11 & 5.2 & \\
\hline Age & & & & \\
\hline & $\geq 70$ & 12 & 7 & 0.334 \\
\hline & $<70$ & 92 & 7.3 & \\
\hline Panc & & & & \\
\hline & Yes & 31 & 7 & 0.319 \\
\hline & No & 73 & 8 & \\
\hline First & & & & \\
\hline & $\leq 4$ months & 19 & 7 & 0.459 \\
\hline & $>4$ months & 85 & 7.3 & \\
\hline Live & & & & \\
\hline & Yes & 72 & 6.5 & 0.054 \\
\hline & No & 32 & 9.9 & \\
\hline CEA & & & & \\
\hline & $\leq 10$ & 69 & 9.9 & $<0.001$ \\
\hline & $>10$ & 35 & 6.2 & \\
\hline CA1s & & & & \\
\hline & $\leq 1,000$ & 57 & 7.5 & 0.621 \\
\hline & $>1,000$ & 47 & 7 & \\
\hline Alb & & & & \\
\hline & $<3.5$ & 40 & 6 & 0.014 \\
\hline & $\geq 3.5$ & 64 & 9.4 & \\
\hline CRP & & & & \\
\hline & $\leq 0.16$ & 35 & 11.6 & $<0.001$ \\
\hline & $>0.16$ & 69 & 5.9 & \\
\hline GPS & & & & \\
\hline & Low (0) & 44 & 10.6 & $<0.001$ \\
\hline & $\operatorname{High}(1,2)$ & 60 & 6 & \\
\hline NLR & & & & \\
\hline & $\leq 5.0$ & 96 & 8.7 & $<0.001$ \\
\hline & $>5.0$ & 8 & 4.6 & \\
\hline
\end{tabular}

Alb albumin, CA19-9 carbohydrate antigen 19-9, CEA carcinoembryonic antigen, CRP C-reactive protein, ECOG PS Eastern Cooperative Oncology Group performance status, GPS Glasgow prognostic score, $N L R$ neutrophil-to-lymphocyte ratio

Table 4 Multivariate analysis of prognostic factors 


\begin{tabular}{cccc}
\hline Variable & HR & $95 \%$ CI & $p$ value \\
\hline GPS $=0$ & 0.47 & $0.29-0.76$ & $<0.002$ \\
\hline $\mathrm{CEA} \leq 10 \mathrm{ng} / \mathrm{mL}$ & 0.47 & $0.29-0.78$ & $<0.003$ \\
\hline
\end{tabular}

$C E A$ carcinoembryonic antigen, $C I$ confidence interval, $H R$ hazard ratio, GPS Glasgow prognostic score

Figures
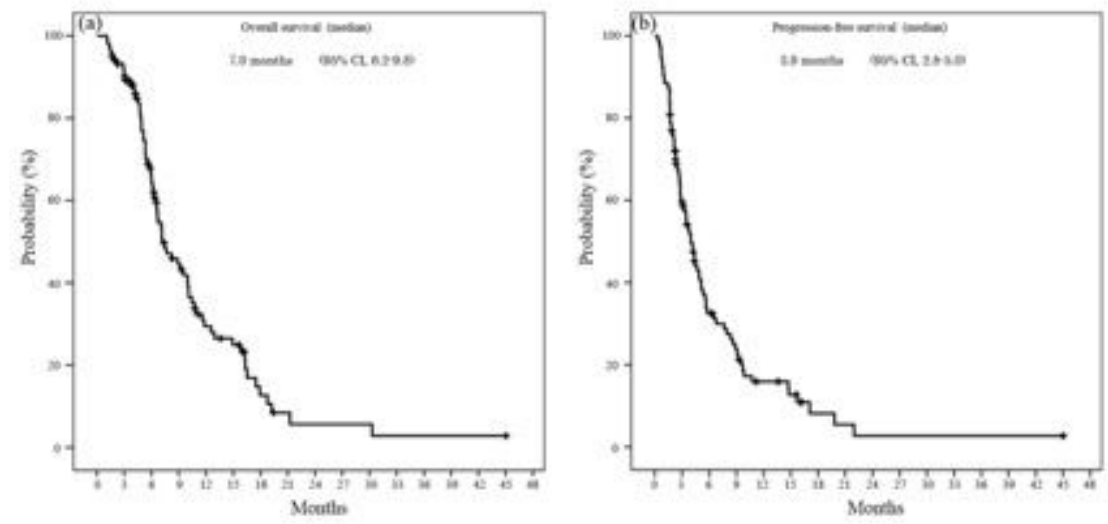

Figure 1

Kaplan-Meier analysis of (a) overall survival and (b) progression-free survival for all patients treated with modified FOLFIRINOX as a second-line therapy following gemcitabine plus nab-paclitaxel. $\mathrm{Cl}$ confidence interval
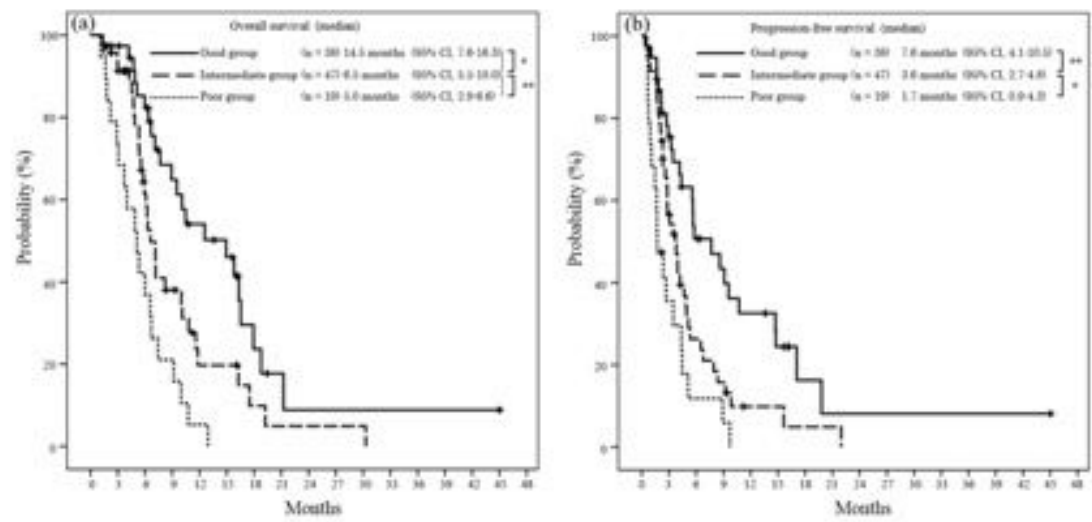

Figure 2

Kaplan-Meier analysis of (a) overall survival and (b) progression-free survival for three subgroups (the poor, intermediate, and good prognostic groups) according to prognostic index using Glasgow prognostic score and carcinoembryonic antigen level. Cl confidence interval. ${ }^{\star} p<0.05,{ }^{\star \star} p<0.01$ 